\title{
Un poète méconnu de Marie Stuart, Lhuillier de Maisonfleur (Réponse à M. Ian R. Morrison)
}

\section{Monia Mezzetti}

\section{(2) OpenEdition}

\section{Journals}

Édition électronique

URL : http://journals.openedition.org/studifrancesi/8157

DOI : 10.4000/studifrancesi.8157

ISSN : 2421-5856

Éditeur

Rosenberg \& Sellier

\section{Édition imprimée}

Date de publication : 1 mai 2009

Pagination : 102-111

ISSN : 0039-2944

\section{Référence électronique}

Monia Mezzetti, « Un poète méconnu de Marie Stuart, Lhuillier de Maisonfleur (Réponse à M. lan R.

Morrison) », Studi Francesi [En ligne], 157 (LIII | I) | 2009, mis en ligne le 30 novembre 2015, consulté le 13 janvier 2021. URL : http://journals.openedition.org/studifrancesi/8157 ; DOI : https://doi.org/

10.4000/studifrancesi.8157

\section{(c) (i) $\odot$}

Studi Francesi è distribuita con Licenza Creative Commons Attribuzione - Non commerciale - Non opere derivate 4.0 Internazionale. 


\section{Un poète méconnu de Marie Stuart, Lhuillier de Maisonfleur (Réponse à M. Ian R. Morrison)}

Dans son article consacré aux rapports entre le concept de Fortune et celui de Providence dans la poésie de Ronsard, Ian R. Morrison démontre d'une manière convaincante, à travers l'étude de deux élégies au sujet de Marie Stuart, qu'en raison de l'«ascendance païenne» ${ }^{1}$ du premier, ce problème est posé avec «une acuité particulière» ${ }^{2}$. Ceci dit, il nous semble intéressant d'approfondir deux points.

Le premier concerne l'interprétation du v. 27 de l'Elegie sur le depart de la royne d'Escosse (incipit: «Comme un beau pré dépoüillé de ses fleurs»), portant sur l'état de Marie Stuart lors de son mariage avec François II: M. Morrison trouve le vers en question («Et d'orpheline ensemble \& estrangere») peu exact, puisqu'à cette époque, soit en 1558, sa mère Marie de Guise était encore vivante (décédée le 10 juin 1560). Selon nous, le terme utilisé par Ronsard doit être mis en relation plutôt avec la perte de son père, le roi d'Écosse Jacques V (1512-1542), qui eut lieu quelques jours après sa naissance ${ }^{3}$. Le mot orphelin peut en effet être utilisé pour indiquer un enfant qui a perdu ses parents, aussi bien que l'un des deux ${ }^{4}$. C'est donc dans cette seconde acception que le mot doit être reçu. Cependant, l'objection de M. Morrison demeure tout à fait légitime à cause du manque, dans le poème, d'autres éléments à même d'envisager le deuil auquel le poète renvoyait.

Deuxièmement, à la fin de son article, M. Morrison soutient que Ronsard ne traite pas toujours Marie Stuart en tant que jouet, sinon victime, de la Fortune ou de la Providence, et, pour le prouver, il cite l'Elegie à H. l'Huillier (incipit: «L'Huillier, si nous perdons cette belle princesse»), également dédiée au départ de Marie Stuart de la France et qui a une allure «assez sereine» 5 .

Nous nous proposons de consacrer quelques mots à ce personnage, Lhuillier ${ }^{6}$,

(1) IAn R. Morrison, Ronsard, la Fortune et la Providence: deux élégies au sujet de Mary Stuart, reine d'Écosse, dans «Studi Francesi», n. 1, janvieravril 2007, pp. 99-106, p. 99.

(2) Ibid.

(3) Jacques $\mathrm{V}$ mourut au château de Falkland (Fife), le 14 décembre 1542, alors que sa fille naquit dans celui de Linlithgow (West Lothian) le 7 ou le 8 décembre. L'incertitude quant à la date de naissance de Marie Stuart dérive des récits des chroniqueurs de l'époque, dont la plupart, comme John Knox, parlent du 8, tandis qu'un seulement du 7; or, celuici, l'évêque John Leslye, avait accès aux documents officiels. On a essayé d'expliquer cette discordance en supposant que, bien que la princesse ait été mise au monde le 7 , la date de l'évènement aurait été dif- férée afin de la faire coïncider avec le jour consacré à la Vierge, dont elle portait le nom (à cet égard, pourtant, une seule source affirme que la princesse avait été baptisée Elizabeth; cfr. Hamilton Papers, éd. J. Bain, Edinburgh, 1890, vol. I, p. 307, cité dans Antonia Fraser, Maria Stuart. La tragedia di una regina, Milano, Mondadori, 1996, p. 20 (Mary Queen of Scots, 1968'). Quant à Marie Stuart, elle crut toujours être née le 8 décembre.

(4) ÉDmond Huguet, Dictionnaire de la langue française du seizième siècle, Paris, Didier, 1961, tome V; ToBler-Lommatzsch, Altfranzösisches Wörterbuch, Wiesbaden, Franz Steiner Verlag, 1965, t. VI.

(5) IAN MORRISON, op. cit., p. 106.

(6) Cfr. infra, p. 108. 
très peu connu - ce qui explique pourquoi M. Morrison n'a ajouté aucune notice, même s'il aurait pu citer son nom complet, étant donné qu'il figure dans l'édition utilisée des Euvres Complètes de Ronsard (éd. Paul Laumonier) - et auteur, lui aussi, d'une élégie sur la reine Marie (B.N.F. ms. 14888 nouv. acq.; incipit: «Ronsard, si nous perdons cette belle princesse» $)^{7}$. Nous essaierons de comparer cette élégie, dans ses traits essentiels, à celle de Ronsard (Elegie à H. l'Huillier; incipit: «L'Huillier, si nous perdons cette belle princesse»).

Malgré le sort de son œuvre, aujourd'hui dans un état fragmentaire et pour la plupart inédite, Lhuillier fut un poète, peut-être pas vraiment célèbre, mais sans doute estimé de ses contemporains ${ }^{8}$ et surtout, selon Brantôme, l'un des trois poètes préférés de Marie Stuart, à côté de ses illustres contemporains, Ronsard et Du Bellay?.

Quant à sa biographie, on ne sait pas grand-chose et le peu de notices se trouvent dans des documents disparates (les Mémoires de Brantôme, des documents officiels, sa correspondance, ses propres poèmes et ceux d'autrui) qui n'éclairent pas tout.

Le Dictionnaire de la Noblesse $e^{10}$ nous apprend (a) qu'il était issu d'une famille, les Lhuillier de Chalandos (ou Chalendeau), originaire du Poitou (b) qu'il était seigneur de Maisonfleur, Bois-Ramort et La Fortelle, des lieux correspondant aujourd'hui, d'après nos recherches personnelles, à des hameaux dans le département de la Seineet-Marne, et finalement (c) qu'il se maria avec Sébastienne d'Alix, mais on ignore tout de sa descendance.

En revanche, on ne peut pas compter sur les notices de La France Protestante ${ }^{11}$ : d'après ce répertoire biographique, «Maisonfleur, dit L'Huillier» pourrait être identifié avec Théodore L'Huillier d'après leur profession commune de capitaine. Cependant, cette hypothèse ne peut absolument pas être prise en considération puisque Théodore vécut après Jérôme et la preuve en est qu'il fut gentilhomme de Henri IV et de Louis XIII.

Cette erreur n'a heureusement pas été répétée dans le Dictionnaire bistorique et généalogique des familles du Poitou ${ }^{12}$, où Jérôme Lhuillier, en tant que parisien, n'est pas mentionné. La seule chose possible est qu'entre les deux il y ait eu un lien de parenté, Théodore ayant été, comme les ancêtres de Jérôme, sieur de Chalendos-en-Brie.

Lhuillier de Maisonfleur entra à la cour comme poète entre 1553 et 1559 mais de toute façon avant 1560 . À cette date en effet, Ronsard le cite, pour la première fois, à propos de «sa chère bande» (à savoir, la Brigade, la future Pléiade) dans Les Isles Fortunées, où son nom («Ici l'Huillier une troupe conduit») ${ }^{13}$ remplace ceux de

(7) À paraître dans «Il Confronto Letterario» (Università di Pavia): Monia MezzetTi, Jérôme Lbuillier de Maisonfleur e l'inedita elegia in onore di Maria Stuarda. Édition critique.

(8) Il est le dédicataire de nombreux poèmes ou recueils d'autres auteurs, comme Brantôme, Ronsard, Desportes. Ses poèmes se trouvent dans plusieurs recueils collectifs. En outre, on compte sept éditions de ses Cantiques (entre 1580 et 1613), le seul ouvrage imprimé.

(9) Pierre de Bourdeilles, seigneur de Brantôme (dorénavant tout simplement BRANTÔME), Discours sur la reyne d'Escosse, jadys reyne de nostre France, dans Recueil des dames, poésies et tombeaux, éd. Étienne Vaucheret, Paris, Gallimard, 1991, pp. 72-73 (dorénavant: Brantôme, op. cit., éd. Étienne
Vaucheret): «Surtout elle aimoit la poësie et les poëtes, mais sur tous Monsieur de Ronsard, Monsieur du Bellay, et Monsieur de Maisonfleur, qui ont faict de belles poësies et ellegies pour elle, et mesme sur son partement de la France, que j'ay veu souvant lire à elle-mesmes [sic] en France et en Escosse, les larmes à l'oeil et les souspirs au coeur».

(10) De La Chenay-Desbois et Badier, Dictionnaire de la Noblesse, Paris, Schlesinger, $1867^{3}$.

(11) Eugène et Émile HaAg, La France Protestante (1846-1859'), Genève, Slatkine Reprints, 1966, VII, pp. 85-87.

(12) Joseph Beauchet-Filleau (éd.), Dictionnaire historique et généalogique des familles du Poitou, Poitiers, $2^{\mathrm{e}}$ éd., 1891-1978, p. 132.

(13) Ronsard, Les Isles Fortunées, v. 71, dans 
Maclou de la Haye et de Jean de Castaigne, qui avaient été rappelés dans l'édition de 1553 («Ici Maclou, là Chataigne conduit») ${ }^{14}$.

Par la suite, il chanta ses poèmes chez les Lorraine ${ }^{15}$, chez Villeroy ${ }^{16}$, il fit partie de l'entourage de Louise de Coligny ${ }^{17}$ puis de Charlotte de Bourbon, à l'époque où elle était la troisième femme de Guillaume d'Orange ${ }^{18}$.

Tout au long de sa vie, Jérôme Lhuillier de Maisonfleur ne se consacra pas seulement à la poésie, mais il travailla à la cour, fut au service de personnages importants, alla à la guerre, entreprit des voyages et séjourna assez longtemps à l'étranger.

Son nom apparaît en effet dans les listes des chevaliers de Henri II et de François $\mathrm{II}^{19}$, des écuyers tranchants ${ }^{20}$, des gentilshommes de chambre ${ }^{21}$ et des échansons $^{22}$ de Catherine de Médicis.

Au début des années soixante, il fut au service du duc François de Guise. En tant que secrétaire ${ }^{23}$, il fut chargé d'une mission à Constantinople ${ }^{24}$, au retour de laquelle il rejoignit son maître à Civitella del Tronto (Italie), en état de siège d'avril à mai $1557^{25}$; ensuite, en raison de sa connaissance de l'espagnol, il entreprit sur sa charge un voyage à Tolède, où il demeura à la cour de Philippe $\mathrm{II}^{26}$. À l'époque des premiers troubles entre catholiques et huguenots, il entra dans l'armée du duc de Guise en qualité de capitaine et il fut, avec Brantôme, parmi les assiégeants d'Orléans (1562-63) ${ }^{27}$.

Ensuite il passa au camp protestant. Selon le témoignage de Brantôme, en 1568, il aida la veuve, elle aussi huguenote, de Fernando de Sanseverino, prince de Salerne (Italie), à faire la quête à la cour de France pour subvenir aux frais d'obsèques de son mari ${ }^{28}$

On ne sait pas exactement s'il se brouilla avec la maison de Lorraine à cause de sa conversion, encore que cela soit vraisemblable; toujours est-il que les membres de cette famille commencèrent à le harceler, allant jusqu'à le faire emprisonner pour avoir fait prêcher dans sa maison et à le faire condamner à mort, ce qui se serait véritablement passé s'il n'eut pas réussi à s'enfuirr' ${ }^{29}$.

Lors du massacre de la Saint-Barthélemy, il se réfugia à l'hôtel de François d'Alençon $^{30}$, qui, au début du mois suivant, le chargea de la négociation de son mariage avec Elizabeth d'Angleterre ${ }^{31}$. Ainsi, il demeura pendant presque une année,

Pierre de Ronsard, Euvres Complètes, éd. Paul Laumonier, Paris, Didier, 1937-68, t. V, p. 179 (dorénavant RONSARD, op. cit., éd. P. Laumonier).

(14) Ibid.

(15) B.N. ms. 1663 fol. $89 \mathrm{r}^{\circ}$.

(16) Ibid.

(17) La Haye, Koninklijke Bibliotheek, ms. 129

A 23, fol.os 40-41, 76-78.

(18) Cfr. dédicace de ses Cantiques.

(19) De La Chenay-Desbois et Badier, op. cit., p. 67; BRAnTôme, Euvres Complètes, éd. Ludovic Lalanne, Paris, Société de l'Histoire de France, 1864-82, t. X, p. 409, note 3.

(20) Ibid.

(21) B.N. Pièces originales, vol. 1772, fol. 189.

(22) Hector DE LA FerRIÈre (éd.), Lettres de Catherine de Médicis, Imprimerie Nationale, Paris 1880-95, supplément, p. 521. Maisonfleur ne recouvrit cette charge qu'en 1572 .

(23) Archives Nationales ms. KK//127 fol.os 2252v.-2253r.; Sonnet de Ronsard: Au Seigneur L'Huillier, incipit: «L'Huillier (à qui Phoebus, comme au seul de nostre age)», dans RONSARD, op. cit., éd. P. Laumonier, t. X, pp. 214, vv. 9-12.

(24) Lettre autographe de Maisonfleur à Eliza- beth d'Angleterre envoyée de Plymouth le 9 avril 1573 (Public Record Office; State Papers 70/127); Sonet a H. Luillier, Seigneur de Maisonfleur, Gentilbomme de leurs magestés, incipit: «Quand Apollon auroit fait un ouvrage», dans RONSARD, op. cit., éd. P. Laumonier, t. XII, p. 145, vv. 6-7.

(25) Lettre autographe de Maisonfleur à Elizabeth d'Angleterre envoyée de Plymouth le 9 avril 1573 (Public Record Office; State Papers 70/127).

(26) Quittance de payement datée 27 octobre 1560: Archives Nationales ms. KK//127 fol.os 2252v.- 2253r.

(27) Brantôme, Euvres Complètes, éd. Prosper MÉrimée et Louis Lacour, Paris, Plon, 1893, t. IX (Rodomontades espaignolles), pp. 28-29.

(28) Brantôme, op. cit., éd. P. Mérimée et L. Lacour, t. II (Vies des grands capitaines estrangers), p. 43.

(29) Lettre autographe de Maisonfleur à Elizabeth d'Angleterre, envoyée de Plymouth le 9 avril 1573 (Public Record Office; State Papers 70/127).

(30) Lettre autographe de Maisonfleur à Charles IX, envoyée de Delft (Pays Bas) le 26 novembre 1573 (B.N. ms. 17199, fol.os 136-141 r.-v.).

(31) Lettre dictée par François d'Alençon à Maisonfleur et adressée à Elizabeth d'Angleterre, 
de septembre 1572 jusqu'à juillet 1573, à la cour anglaise, où il entra en contact avec la reine en personne, son chancelier Lord Burghley et son favori, le comte Robert Leicester.

En avril 1573, il participa à l'expédition infructueuse du duc de Montgomery en aide aux protestants assiégés à La Rochelle ${ }^{32}$.

À partir de juillet 1573, croyant avoir achevé sa mission en faveur du duc d'Alençon, il abandonna l'Angleterre et se rendit en Hollande, à la tête d'une compagnie d'hommes de pied huguenots pour combattre contre les Espagnols ${ }^{33}$.

C'est probablement au cour de cette entreprise qu'il décéda, mortellement frappé par l'un de ses soldats au cours d'un duel ${ }^{34}$.

De toute façon, son décès eut lieu avant 1580, car cette année-là Jacques Heinrick d'Anvers publia la première édition posthume de ses Cantiques ${ }^{35}$.

De la bibliographie de Lhuillier de Maisonfleur, on connaît:

- B.N.F. ms. 1663, fol.89 (incipit: «Tout ce qui prend en ce monde naissance») et fol. 122 (incipit: «Dieu qui nasquis d'une mere pucelle», «Ce qui faict plus juger une ame estre immortelle») (trois pièces inédites);

- B.N.F. ms. 11688 nouv.acq., fol. ${ }^{\text {os }}$ 1-6 r.-v., Elegie faicte par m. Luyllier sur le depart de la royne Marie (inédite);

- Cantiques (éd. 1580, dont on ne possède aucun exemplaire, '81,'84,'92, 1603, '13, dont plusieurs exemplaires sont conservés à la Bibliothèque Nationale);

- Koninklijke Bibliotheek de La Haye, ms. 129 A 23 fol.os 40-41 (pièce inédite; incipit: «Le soleil qui fait son seiour»); fol.o 76-78 (Cantique faict à la Sainct Barthélemie, publié dans Le Chansonnier Huguenot du XVI siècle ${ }^{36}$ et par Mario Richter ${ }^{37}$, qui, à son tour, a reproduit le texte établi par $\mathrm{H}$. Bordier);

- B.N.F. ms. 17199 fol. 136-141 (une lettre à Charles IX) (la première page a été partiellement publiée par Jacques Lavaud $\left.{ }^{38}\right)$;

- sa correspondance avec François d'Alençon, Elisabeth Tudor et Lord Burghley, conservée en partie au Record Office de Richmond et en partie à Hatfield House, l'ancienne résidence du chancelier (partiellement éditée par Hector de la Ferrière, mais avec de nombreuses, et quelquefois inexplicables, erreurs de transcription de mots et de dates, et en outre avec quelques fautes quant à l'indication de la localisation des documents) $)^{39}$.

envoyée de Paris le 23 septembre 1572 (Hatfield House; Cecil Papers 148/92).

(32) Lettre autographe de Maisonfleur à Elizabeth d'Angleterre (Public Record Office, State Papers 70/127).

(33) Lettre autographe de Maisonfleur à Lord Burghley, envoyée de Londres le 13 juillet 1573 (Public Record Office, State Papers 70/128); Lettre autographe de Maisonfleur à Charles IX, envoyée de Delft (Pays Bas) le 26 novembre 1573 (B.N. ms. 17199), où toutefois il ne parle pas de sa mission en Angleterre en faveur du duc d'Alençon.

(34) Brantôme, op. cit., éd. P. Mérimée et L. Lacour, t. VIII (Discours sur les duels), pp. 202-203.

(35) Claude-Pierre Goujet, Bibliothèque Françoise ou Histoire de la Littérature française (1750), Slatkine Reprints, Genève, 1966, p. 14; Les Bibliothèques Françaises de La Croix du Maine et de Du Verdier sieur de Vauprivas; nouvelle édition dédiée au roi ..., Paris, chez Michel Lambert, 1772-1773.

(36) Henri LéONARD Bordier, Le Chansonnier Huguenot du XVI siècle (Paris-Lyon, 1870), Genève, Slatkine Reprints, 1969, pp. 288-294.

(37) Mario Richter, La poesia lirica in Francia nel secolo XVI, Milano-Varese, Istituto Editoriale Cisalpino, 1971.

(38) JaCQues LaVAud, Un poète de cour au temps des derniers Valois: Philippe Desportes (1546-1606), Paris, Droz, 1936, pp. 124-125, note 2.

(39) Hector de La Ferrière, Le XVI ie siècle et les Valois d'après les documents inédits du British Museum et du Record Office, Paris, Imprimerie Nationale, 1879 , pp. 337-390. Quant aux inexactitudes de La Ferrière, nous nous permettons de renvoyer à notre article Il carteggio di Lbuillier de Maisonfleur 
Brantôme ne cite jamais le nom complet du gentilhomme, mais l'appelle Lhuillier (ou Luyllier, dans le titre de l'élégie sur le départ de Marie Stuart) ou Maison[-]fleur, et dans ses Mémoires, il mentionne deux fois un Maisonfleur catholique et deux autres fois un personnage homonyme mais huguenot; c'est pour ces raisons que le principal spécialiste du mémorialiste, Étienne Vaucheret, a douté qu'il pût s'agir de la même personne ${ }^{40}$.

Il est donc évident que la première difficulté concerne son nom. Or, c'est grâce à Ronsard qu'il est possible de le connaître dans sa forme complète. Les outils officiels, comme le catalogue papier ${ }^{41}$ et celui informatisé de la Bibliothèque Nationale de France, ne sont en effet d'aucune aide en ce cas, puisque le personnage n'est pas enregistré sous le nom de L'Huillier mais, comme le voulait l'usage de l'époque, sous celui d'une de ses dépendances, Maisonfleur (en Brie, dont il était sieur...) ${ }^{42}$. De surcrô̂t, à la suite d'une ancienne erreur de Du Verdier ${ }^{43}$, déjà signalée par Jacques $\mathrm{Lavaud}^{44}$ et par Paul Laumonier ${ }^{45}$, on lui a attribué le prénom d'Etienne, alors qu'il s'appelait Jérôme.

Peu importe que Ronsard en personne ou ses secrétaires aient écrit le nom du personnage en utilisant une orthographe variable, car les changements n'impliquent aucune différence au niveau de la prononciation, et cela vaut pour Lhuillier (autres formes: Luyllier, Luillier, L/l'Huillier, L'Huilier) aussi bien que pour Maisonfleur (autres formes: Maison-Fleur, Maison Fleur, Maysonfleur) ${ }^{46}$. Ce qui importe c'est de découvrir que, dans les documents anciens, son nom apparaît dans sa forme complète et exacte, c'est-à-dire Lhuillier de Maisonfleur.

On lit en effet cette dénomination:

(a) dans le titre du sonnet avec lequel Ronsard dédicaça au gentilhomme le Second Livre des Nouvelles poésies (1564) (Sonet a H. Luillier, Seigneur de Maisonfleur, gentilhomme servant de leurs magestés; incipit: «Quand Apollon auroit fait un ouvrage» $)^{47}$;

(b) dans celui de l'élégie concernant le départ de la reine Marie pour l'Écosse, publiée en 1564 (Elegie a H. L'Huillier, Seigneur de Maisonfleur; incipit: «L'Huillier, si nous perdons cette belle princesse»). Par la suite, le titre changea tout simplement en Elegie (éditions de 1567, 1578 et 1584), alors qu'en 1571 il devint Elegie sur le départ de la royne d'Escosse, qui malheureusement est identique au titre du poème dont l'incipit est «Comme un beau pré dépoüillé de ses fleurs» (et c'est peut-être pour cela qu'il ne fut pas répété dans les éditions suivantes du même recueil, les Nouvelles poé-

risalente agli anni 1572-1573, dans «Il Confronto Letterario», n. 41, 2004, pp. 35-62.

(40) BRAnTôme, op. cit., éd. É. Vaucheret, pp. 1106 et 1479 .

(41) C'est-à-dire le catalogue consultable sur place et le Catalogue Général des livres imprimés de la Bibliothèque Nationale, Paris, Paul Catin Editeur (1960-1969), t. XXVI.

(42) Nos recherches nous ont permis de localiser Maisonfleur dans la commune de Pommeuse (dép. de Seine-et-Marne), où personne ne connaît l'auteur et où il n'existe ni château ni ruines portant ce nom. De nos jours, donc, Maisonfleur est la dénomination d'un endroit actuellement identifiable grâce au nom de la rue dont il est l'éponyme. Quant aux autres fiefs de Jérôme Lhuillier, tous les deux se trouvent dans l'ancienne Brie: BoisRamort (commune de Chevry-en-Serenne; dép. de Seine-et-Marne) est un hameau où un ancien mo- nastère, aujourd'hui utilisé comme ferme, constitue les seules vestiges du passé; La Fortelle (commune de Nesle; dép. de Seine-et-Marne) est elle aussi un hameau où autrefois il existait un château qui a été détruit à la Révolution.

(43) Du VerdiER, Bibliothèque Françoise, 1772, t. III, pp. 517-518.

(44) JacQues LaVAud, op. cit., pp. 124-125, note 2.

(45) Ronsard, op. cit., éd. P. Laumonier, t. X, p. 213 , note 1.

(46) Pour ces variantes nous nous permettons de renvoyer à notre article Precisazioni su Jérôme Lbuillier de Maisonfleur, gentiluomo e poeta tra ultimo Rinascimento e conflitti religiosi, dans «Quaderni del Dipartimento di Lingue e Letterature Neolatine, Università degli Studi di Bergamo», 2001, pp. 7-31.

(47) Ronsard, op. cit., éd. P. Laumonier, t. XII, pp. $145-146$. 
sies); finalement en 1587 le titre est complètement différent et semble faire allusion à la mort du dédicataire (Regret à l'Huillier parisien, pour elle-mesme [= la même]);

(c) dans le B.N.F. ms. 842 (terminus ante quem 1569), contenant une version de l'Orlando Furioso et où il y a la dédicace de Philippe Desportes à «Jérôme Lhuillier, sieur de Maisonfleur» ${ }^{48}$.

La dédicace du ms. 842 est très intéressante, au moins à deux égards.

D'abord parce qu'elle révèle le prénom du gentilhomme, autrement jamais mentionné (Brantôme, tout en s'adressant à lui d'un ton affectueux, l'appelle «l'Huillier» dans ses poèmes et «Maisonfleur» dans ses Mémoires) ou celé dans les poèmes de Ronsard sous la lettre «H.», l'initiale du prénom Herosme (autre forme pour Jérôme ou Jherosme).

En dehors du domaine littéraire, le véritable nom de baptême du gentilhomme apparaît dans un document des Archives Nationales de Paris (ms. KK//127), correspondant à une quittance de payement de 1560, où «Jherosme Lhuillier [...] secretaire de Monseigneur le duc de Guyse» ${ }^{49}$ est indemnisé du voyage effectué en 1560 sur chevaux de poste de Blois à Tolède.

L'autre raison pour laquelle le ms. 842 doit retenir notre attention est liée à la modification de la dédicace, qui en 1572, lors de la publication du livre, est adressée à Charles IX. L'hypothèse concernant la substitution du nom d'un simple gentilhomme par celui du roi ne saurait nous satisfaire et doit donc être écartée. S'il en était ainsi, n'importe quelle dédicace, de Desportes ou d'autres poètes, aurait en effet dû être changée et, à la limite, être modifiée à l'arrivée des nouveaux souverains (à savoir, Henri III en 1573 et Henri IV en 1589), ce qui est inconcevable. À l'origine de la conduite de Desportes, il doit y avoir un tout autre motif, qui explique aussi les modifications analogues apportées par Ronsard, à savoir la conversion du gentilhomme au calvinisme (survenue, sur la base de l'étude des variantes dans les dédicaces et à l'intérieur des poèmes, vers 1567).

Seul Brantôme ne modifia pas ses vers, n'oublia pas de citer Lhuillier de Maisonfleur dans ses Mémoires ni, heureusement, ne se débarrassa de son élégie sur Marie Stuart. Tout au contraire, il la garda ou en fit recopier le texte ${ }^{50}$ (après en avoir peutêtre gardé une copie manuscrite) dans un volume contenant, dans la première partie, les poèmes d'autrui [Recueil de poésies diverses], et dans la seconde les siens (Recueil d'aulcunes rymes de mes jeunes amours).

(48) B.N. ms. 842, fol. $72 \mathrm{v}$ $2253 \mathrm{r}$.

(49) Archives Nationales, KK//127 fol.os 2252v.-

(50) Cette incertitude dépend du fait que l'on ignore la date de composition du manuscrit. Évidemment, il est possible que Brantôme l'ait fait commencer à l'époque du départ de Marie Stuart. $\hat{A}$ notre avis, le sujet du poème en question pourrait être considéré comme le terminus ad quem ou le terminus post quem. Cependant, une autre hypothèse serait valable, à savoir que le manuscrit fût composé pendant la longue période de repos forcé, provoqué par une chute de cheval, durant laquelle Brantôme dicta ses Mémoires. La bipartition du ms. 11688 nowv. acq. constitue en effet le résultat d'un triage, à l'occasion duquel Brantôme aurait pu se servir de manuscrits originaux: quant à l'élégie de Maisonfleur, nous ne savons et on ne saura jamais dire si le copiste a utilisé un texte autographe ou plutôt une copie, car, jusqu'à présent, aucun exemplaire n'a été retrouvé. 
On pourrait attribuer la fidélité de Brantôme au fait que ses poèmes ne furent pas publiés et donc que son lien compromettant avec Maisonfleur ne pouvait pas devenir de notoriété publique. Cette explication se heurte pourtant à notre ignorance quant au renom de leur amitié.

Quoi qu'il en soit, on peut interpréter le choix de Brantôme de garder (a) le nom de l'auteur, «Luyllier», dans le titre, et (b) l'élégie (qu'il aurait pu ne pas faire recopier, mais aussi détruire, par exemple en l'enlevant du manuscrit, au cas où celui-ci aurait déjà été composé), comme un geste, sinon d'amitié, du moins de dévouement. En effet, non seulement Brantôme ne voulut pas oblitérer le nom de Lhuillier dans ses propres vers, mais il plaça l'élégie au début du manuscrit, dans les six premières pages, en tête des autres vers, donc à une place d'honneur.

Le privilège accordé par Brantôme à Lhuillier de Maisonfleur n'est pas seulement témoigné par ce positionnement, mais aussi par l'éloge qu'il en fait dans ses Mémoires («Monsieur de Maisonfleur, gentil cavallier pour les lettres et pour les armes, en fist pour ce subject une fort belle ellegie» ${ }^{51}$ ) et par l'ouvrage qui suit l'élégie (fol. 6), anonyme et qu'on pourrait attribuer au même Brantôme:

Ronsard, qui aultrefoys d'un vol audacieux

S'est poulcé jusqu'au Ciel sur la lire sonante,

Pleurant trop le deppart d'une Royne excelante,

Fut changé en ce roch par le vouloir des Dieulx;

Et Lhuillier, son nepveu, par ce depart piteulx

Remplissoit tout le ciel d'une voix gemissante,

Quand, par pityé divine, en ceste onde courante

Son corps fut transformé, rendant son ame aux Cieulx.

De mortelz qu'ilz estoient, or ilz sont insensibles

Et ne sentent non plus que pierres impassibles.

Las! Et qui le croyroit si l'on n'y venoit veoir.

Au moings chascun passant le Ciel priera son tour,

Que si jamais la Royne en France faict retour,

Ilz leur rendent leurs yeulx pour la venir revoir ${ }^{52}$.

Dans ces vers, on avoue que l'auteur du poème précédent (à savoir l'élégie de «Luyllier») a «mieux fait» que Ronsard, dont il serait le «neveu». Il va sans dire que ce terme vise seulement à rendre hommage à la stature de Ronsard, qui de son côté était bien conscient de sa valeur (il arriva à exprimer son orgueil fou dans le fameux vers: «Je suis Ronsard, que cela te suffise»).

À la rigueur, cet éloge est doté (a) d'une valeur générale, visant à soutenir qu'avec cette élégie «Luyllier» a devancé l'œuvre de Ronsard, ou (b) d'une valeur spécifique, donc que cette élégie est meilleure qu'un autre poème de Ronsard sur le même sujet.

(51) BRantôme, op. cit., éd. É. Vaucheret, p. 79 (Discours sur la reyne d'Escosse, jadys reyne de nostre France).

(52) Selon la notice d'Étienne Vaucheret (BranTÔME, op. cit., éd. É. Vaucheret, p. 1479), les éditeurs précédents de ce recueil, le docteur Galy et Perceau, n'ont pas retenu ces stances en vers oc- tosyllabes comme étant de Brantôme et ils n'ont proposé aucune attribution. Vaucheret non plus, même s'il transcrit le texte (BRANTÔME, op. cit., éd. É. Vaucheret, p. 728), où, selon lui, le poète s'exprime au nom d'une jeune femme. Une hypothèse que nous ne partageons pas en raison du manque d'éléments probants. 
Or, il s'agit précisément de l'Elegie a H. L'Huillier, Seigneur de Maisonfleur, dont l'incipit «L'Huillier, si nous perdons cette belle princesse» a été repris par Maisonfleur, qui substitua son propre nom par celui, toujours dissyllabique, de Ronsard: «Ronsard, si nous perdons cette belle princesse».

Le poème anonyme du B.N. ms. 11688 nouv.acq. fol. 6 v. n'est pas la seule preuve que Maisonfleur aurait composé son élégie après Ronsard.

Que son poème soit une véritable réponse à celui de Ronsard apparaît dans les derniers vers de l'élégie même, où, malgré les nombreuses erreurs (lacunes de vers, vers hypomètres, orthographe incorrecte), on parvient à lire une déclaration de Maisonfleur très claire à ce sujet: «Or desia tu m'as dict qu'un rocher tu veulx estre» (v. 311). À moins de supposer qu'à l'origine il y ait eu une conversation entre les deux poètes, on peut légitimement penser que l'aspiration à la métamorphose spéléologique à laquelle Maisonfleur répond par la sienne, aquatique, est exactement celle exposée par Ronsard dans l'Elegie a H. L'Huillier, Seigneur de Maisonfleur («Le jour que je voyray son depart aprocher, I Je veux, pour ne le voir, devenir un rocher, I Sourd, muet, insensible [... $\left.]^{53}\right)$. Un poème, publié en 1564 , mais qui sans aucun doute circulait à la cour sous forme manuscrite et que vraisemblablement Maisonfleur connaissait, d'autant plus qu'il en était le dédicataire.

D'autres vers de Maisonfleur éclairent du reste l'époque de composition de son poème et par conséquent de celui de Ronsard. Dans sa description de l'immense chagrin éprouvé par la Nature à la nouvelle du départ de la reine, Maisonfleur cite les mois de mars et d'avril. Or ceci coïncide exactement avec l'historiographie au sujet des projets de Catherine de Médicis et de la cour de France quant à l'avenir de la jeune reine, qui, comme M. Morrison l'a rappelé, était «Et d'orpheline ensemble \& estrangere». Puisque son départ ne fut pas décidé à la dernière minute, mais au printemps 1561, on peut supposer aussi que les deux élégies furent composées avant cet événement et que, de ce fait, elles faisaient partie de ce corpus (c'est là une hypothèse basée aussi sur le témoignage de Brantôme, selon lequel Marie Stuart emporta avec elle les ouvrages de ses poètes préférés $)^{54}$.

L'élégie de Maisonfleur est assez différente de celle de Ronsard.

Premièrement, en ce qui concerne la longueur: le poème de Ronsard compte 74 vers, celui de Maisonfleur à peu près 332; le texte présente en fait des lacunes, et, d'après notre transcription, il semble qu'il manque quatre vers (vv. 267-268; 325; 327), dont le positionnement, dans la partie finale du poème, autorise à attribuer la cause à une baisse typique du niveau d'attention du copiste.

Quant à l'imaginaire littéraire, chez Ronsard l'emploi de la mythologie est assez étendu, tandis que chez Maisonfleur sa présence est plus discrète.

On a l'impression que Ronsard se plaît à citer les mythes, puisqu'il les énumère d'une façon presque paroxystique; on reconnaît dans l'ordre celui d'Aurore et Thiton (v. 10: «Quand Thiton en ses bras tient sa femme couchée»), ayant pour but la formulation d'une périphrase signifiant que l'Aurore tarde à se montrer ${ }^{55}$, mais aussi ceux d'Adonis (v. 21: «Et les roses qui sont du sang d'Adonis teintes»), d'Hélène,

(53) Ronsard, op. cit., éd. P. Laumonier, p. 191, t. XII, vv. 35-37. En réalité, l'hyperbole se poursuit avec l'image du rocher transformé en eau de fontaine et avec une troisième métamorphose du poète en oiseau pour pouvoir continuer à admirer le visage de la reine en voyage pour l'Écosse.
(54) Brantôme, op. cit., éd. É. Vaucheret, p. 73. (Discours sur la reyne d'Escosse, jadys reyne de nostre France).

(55) Cfr. Paul Laumonier in Ronsard, op. cit., éd. P. Laumonier, t. XII, p. 190, note 1. 
lorsqu'elle est confiée par Pâris au roi Protée (vv. 27-30: «Si son idolle feinte au moins nous demouroit, I En s'en allant de nous toute ne s'en iroit, I Et aurions le plaisir du sage Roy Prothée | Qui d'Helene retint la figure empruntée»), de Jupiter et Europe (v. 59: «Car celle que porta le Boeuf Sydonien»), et encore de Jupiter et Léda (v. 60: «[Celle] Que le Cygne trompa [...]») ${ }^{56}$. Cela, comme on vient de le dire, le long d'un poème de moins de quatre-vingt vers.

$\mathrm{Au}$ contraire, les personnages mythologiques sont rarement cités sans référence à des événements fictifs spécifiques: les Grâces sont évoquées tout simplement en tant que «les trois filles des cieux» (v. 24), qui se chargent d'orner Marie Stuart «de leurs dons les plus beaux» (v. 25) et qui, pour «mieux la servir» (v. 26), abandonnent les cieux; les Nymphes sont rappelées par une similitude où le poète compare sa souffrance à la leur, lorsqu'elles assistent, impuissantes, au délabrement de ce dont elles sont les protectrices, à savoir les fleurs enlevées par le vent, les feuilles des arbres, les fontaines souillées et embourbées (vv. 38-42) s. $^{57}$.

Chez Maisonfleur, il n'y aucune réminiscence des mythes, mais tout simplement un usage des personnages en fonction de leur identité, ce qui permet au poète d'en citer un plus grand nombre, sans pour cela produire cet effet de comble que l'on ressent dans le poème de Ronsard.

Le traitement des personnages, quoique analogue aux deux derniers exemples tirés de l'Elegie à $H$. l'Huillier, présente la particularité de joindre les créatures mythologiques à la représentation (a) du regret pour le départ de la reine ou (b) du temps jadis, qui était heureux parce que correspondant au séjour de la dame à la cour de France. De ce fait, les êtres mythologiques apparaissent le long du texte, non pas en arrière plan, mais en tant que protagonistes du récit.

Alors que Ronsard se limite à imaginer les Grâces au travail auprès de Marie Stuart pour en faire une «princesse | qui en un corps mortel ressemble une déesse» (Elegie à H. L'Huillier, vv. 1-2), Maisonfleur fait de Flore et Zéphyr les serviteurs du Printemps, qui, à cause de leur affliction pour la perte de la dame, refusent, comme le reste de la Nature, de collaborer à son retour, de sorte que le pays se trouve dans un hiver perpétuel. D'autres personnages mythologiques participent au deuil du Printemps: Hélicon, Olympe, Flore, Pomone, Écho, Pégase, les Muses.

Dans l'élégie de Ronsard, il n'y a qu'un seul et rapide aperçu du bonheur qui régnait en France avant le départ de la reine; le mot clé est l'adverbe «toujours» et la description du pouvoir thaumaturgique de la beauté de la reine concerne non pas le passé, lors de son séjour en France, mais son avenir en Écosse:

Toujours de soubs ses pieds la terre se peindra

D'un beau tapis de fleurs, les eaux seront paisibles.

Les vens apaiseront leurs alaines terribles,

La mer se fera douce, \& pour voir sa beauté

Le soleil espendra sur elle sa clartée $e^{58}$.

Au contraire, dans le poème de Maisonfleur, on assiste à une amplification de cette phase, de sorte que, compte tenu du nombre des vers, le tableau de la gaieté est complémentaire de celui du deuil. Le poète consacre en effet au cadre joyeux $44,8 \%$

(56) RonsARd, Elegie à H. Lbuillier, seigneur de Maisonfleur, dans RONSARD, op. cit., éd. P. Laumonier, t. XII, pp. 190-191

(57) Ibid.
(58) Ronsard, Elegie à H. L'Huillier, dans RonSARD, op. cit., éd. P. Laumonier, t. XII, p. 189, vv. 68-72. 
de ses vers (soit cent quarante-neuf vers - de v. 89 à v. 238) et à celui du chagrin généralisé $54,5 \%$ (soit cent quatre-vingt-un - de v. 1 à v. 88, puis de v. 239 à v. 332).

Le pivot de la représentation de la phase de bonheur est constitué par l'amour tendre du Printemps pour Marie Stuart. Ce détail est inédit chez Ronsard, qui fait allusion à cette saison erronément en tant que période où Marie Stuart serait née ${ }^{59}$ («Au millieu du Printemps, entre le Lys naquit I Son corps, qui de blancheur les Lys mesmes veinquit» $\left.{ }^{60}\right)$.

Stimulé peut-être par cette erreur de Ronsard, Maisonfleur choisit d'attribuer une tout autre valeur au Printemps. Il en fait, comme on vient de le dire, la saison amoureuse de Marie Stuart, ce qui lui donne l'occasion d'accroître une partie du poème. L'enrichissement est en fait obtenu par (a) l'introduction de nombreux personnages mythologiques et (b) l'ajout d'une longue similitude ayant pour protagoniste un homme amoureux de sa dame, qui, comme le Printemps, se soucie de sa propre toilette et éprouve des sentiments de gaieté ou de tristesse, selon qu'il peut ou non la rencontrer.

Parmi les êtres mythologiques participant à la cour faite par le Printemps à Marie Stuart, le poète cite: Écho, qui répétait dans les alentours les paroles d'amour du Printemps; Zéphyr qui, avant de défendre son arrivée, versait des roses vermeilles dans les lieux de leurs rencontres; Pomone, qui favorisait le mûrissement des fruits; les dryades, les naïades, les satyres et les faunes, qui fréquentaient les mêmes endroits et qui égayaient le Printemps; les nymphes et les Muses, qui, après avoir réalisé des guirlandes de fleurs, commençaient à danser au son de la lyre d’Apollon.

Ainsi, au lieu de rappeler les récits des Anciens, Maisonfleur mentionne les êtres mythologiques en fonction de leurs prérogatives, de sorte qu'ils contribuent à la représentation d'un tableau d'affliction.

En définitive, donc, la mythologie est subordonnée au projet poétique de l'auteur, où ce type de personnages aussi bien que les éléments naturels personnifiés sont plutôt des hypostases du bonheur ou de la souffrance. De cette manière, dans le poème on peut distinguer trois domaines homologues à même d'évoquer le regret suscité par le départ de la reine: la cour, en proie au chagrin, la Nature paralysée par sa propre inertie, les divinités et les personnages mythologiques atteints par un changement drastique de leurs habitudes.

Dans cet état généralisé de paralysie et d'accablement, il n'y a aucune place pour des réflexions sur la Fortune ou la Providence. De même que dans l'Elegie à $H$. Lhuillier, ce thème n'est pas évoqué, ce qui démontre, compte tenu aussi des autres poèmes connus de Maisonfleur, que le poète n'était pas intéressé par ce genre de sujet.

Enfin son élégie, en tant que poème lyrique exprimant des sentiments mélancoliques, exclut d'envisager le sort de Marie Stuart. Le poème reste ancré au présent, accablé par la douleur, et le regard du poète ne dépasse pas les confins de la France. Contrairement à Ronsard qui, à la fin du poème, décrit les effets positifs de la présence de la jeune femme en Écosse, Maisonfleur récuse ce dénouement. Au lieu de traverser la Manche, l'image de Marie Stuart reste en France, en tant que symbole de beauté et souvenir d'une créature bénéfique.

MONIA MEZZETTI

(59) Cfr. supra note 3.

(60) Ronsard, Elegie à H. L'Huillier, dans Ron-

SARD., op. cit., éd. P. Laumonier, t. XII, p. 190, vv. 19-20. 